\begin{tabular}{|c|c|c|}
\hline Beitr. Ent. & Keltern & ISSN 0005 - 805X \\
\hline $\mathbf{5 1}(2001) 2$ & S. $401-409$ & 16.12 .2001 \\
\hline
\end{tabular}

\title{
Lebensdaten und Heteroptera-Sammlung des FELIX vON BAERENSPRUNG (1822-1864)
}

Mit 3 Figuren

JÜRGEN DECKERT

Zusammenfassung

Felix von Baerensprung lebte von 1822 bis 1864 . Et beschäftigte sich nebenbei mit der Entomologie und war Atzt in Halle und Betlin. Einige mitteleuropäische Heteroptera sind von ihm beschrieben worden, außerdem sammelte er in Berlin und Brandenburg in den fünfziger Jahren des 19. Jahrhunderts. Neben Angaben zu seinen Lebensdaten werden von ihm beschriebene Arten, die vorhandenen Typen in der Sammlung des Museums für Naturkunde und seine Veröffentlichungen über Heteroptera aufgelistet.

Für Graphosoma oculatum, Alydus sareptanus und Antbocoris elegantulus werden Lectotypen designiert.

\section{Summary}

The German physician Felix von Baetensprung (1822-1864) lived in Halle and Berlin. He was a medical doctor and made entomological studies only additionally. Baerensprung described Heteroptera from Central Europe and collected also in the fiftieth of the 19th century in Berlin and Brandenburg. Data on his life were summarised. A list of the Heteroptera species he described and as his publications on Heteroptera are listed. This includes a list of types housed in the collection of the Museum of Natural History in Berlin. Lectotypes are designated for Graphosomo oculatum, Alydus sareptanus and Anthocoris elegantulus.

\section{Key Words}

Heteroptera, Baerensprung, curriculum vitae, type specimens, lectotype designation, publication list.

\section{Einleitung}

Felix von Baerensprung ${ }^{1}$ ist allen Entomologen, die sich mit der mitteleuropäischen Heteroptera-Fauna beschäftigen, als Autor von Artbeschreibungen ein Begriff. Die Gattung Myrmedobia ist von Baerensprung aufgestellt worden und auch das Taxon Bryocorinae (Miridae) geht auf Baerensprung zurück, er hat in seinem „Catalogus Hemipterorum Europae“ zum ersten Mal diese Gruppierung als Tribus Bryocorides verwendet.

\footnotetext{
${ }^{1}$ Es sind zwei verschiedene Schreibweisen seines Namens überliefert: "Baerensprung” oder „Bärensprung” Da trotz der Verwendung des Vokales, , $\ddot{a}$ an anderer Stelle in den gedruckten Artikeln immer die Schreibweise „Baerensprung" verwendet worden ist, habe ich diese übernommen.
} 
Es ist zwar bekannt, dass sich seine Sammlung einschließlich des Typenmaterials im Berliner Museum für Naturkunde befindet, jedoch sind bis auf sein Todesjahr (1864) keine persönlichen Daten allgemein bekannt (HORN et al. 1990).

Baerensprung war ein Mensch mit herausragender Vielseitigkeit. Er betrieb seine entomologischen Studien nur nebenbei. Er starb 44-jährig und ein Nachruf auf ihn, der in den Annalen des Charité-Krankenhauses im Band XII veröffentlicht worden ist, blieb den Entomologen offensichtlich unbekannt. Diesem Nachruf sind die folgenden Informationen über die Lebensdaten Baerensprungs entnommen. Außerdem erschien ein kurzer Nachruf in der Berliner Entomologischen Zeitschrift von STEIN (1864).

\section{Lebensdaten des Felix von Baerensprung}

Friedrich Wilhelm Felix von Baerensprung wurde atn 30. März 1822 als Sohn des damaligen Oberbürgermeisters von Berlin geboren. Ab 1840 studierte er naturwissenschaftliche Fächet - vor allem Entomologie - in Berlin und ab 1843 hörte er Vorlesungen über Innere Medizin in Halle. Nach seinem Staatsexamen studierte er außerdem 4 Monate Pathologische Anatomie in Prag. Er war schon vor seinem Staatsexamen Assistent in einer Klinik für Innere Medizin in Halle und war zwischen 1845 und 1847 dort erster Assistenzarzt. In dieser Zeit habilitierte er sich mit einem medizinischen Thema über Hautkrankheiten. Nach einer kurzen Zeit als Leiter eines Kinderlazaretts ging er 1848 zurück an die Klinik in Halle. 1850 heiratete er Marie Bluhne, ließ sich in Halle als Arzt nieder und gründete eine medizinische Privatklinik. Nebenbei hielt er Vorlesungen und publizierte sowohl auf medizinischem als auch auf entomologischem Gebiet.

Im Jahr 1853 folgte Baerensprung einem Ruf an die Berliner Charité als "dirigirender" Arzt und wurde dort drei Jahre später Außerordentlicher Professor. Sein besonderes Interesse galt Hautkrankheiten und der Syphilis. Seine zahlreichen medizinischen Arbeiten machten ihn zu einem anerkannten Spezialisten auf diesem Gebiet. Er war übrigens der erste, der die Temperaturmessung bei Kranken als notwendigen Standard wissenschaftlich begründet und eingeführt hat. Sein Ziel, an der Spitze einer großen deutschen medizinischen Klinik zu stehen, erreichte er nicht. Neben richtungsweisenden Arbeiten waren einige seiner Arbeiten sehr umstritten und offensichtlich "mehr anregend als aufklärend" (VEIT 1864). Ab 1860 etwa wurde er zunehmen von der schweren Krankheit Syphilis gezeichnet, die sich in einer schleichenden Persönlichkeitsänderung in zunehmender Exzentrizität und Schroffheit seines Verhaltens äußerte und schließlich in einer Dementia paralytica endete. Seine Laufbahn wurde so tragischerweise in einer Nervenklinik bei Kiel beendet. Bei einem Spaziergang in Kiel im Jahr 1864 stürzte er von einem Steg ins Meer und ertrank. 


\section{Die entomologische Sammlung von Baerensprung}

Durch Vermittlung von Gustav Kraatz (1831-1909), dem Stifter des Deutschen Entomologischen Institutes, schenkte die Witwe Baerensprungs seine Sammlung dem zoologischen Sammlung des Königlichen Museums. Die Sammlung Baerensprung umfasst die von ihm beschriebenen Arten und seine in Berlin und Brandenburg gesammelte Wanzen. Baerensprungs umfangreiche Funde aus Berlin und Brandenburg aus der Zeit zwischen 1853 und etwa 1860 wurden von SCHUMACHER (1912a, b) ausgewertet. Die von Baerensprung bei Halle gesammelten Wanzen listete SCHUMACHER (1913) auf.

$\mathrm{Zu}$ seiner Sammlung gehört ausschließlich europäisches Material, insgesamt sind es etwa 850 Arten, davon 350 aus der Berliner Gegend (STEIN 1864). Es gehören dazu auch in Berlin und der Umgebung selten nachgewiesene Arten, wie zum Beispiel Cryptostemma alienum HERRICH-SCHAEFFER, Sigara bellensii C. SAHLBERG oder Eysarcoris fabricii KIRKALDY. Baerensprung bemühte sich um eine möglichst vollständige Kollektion von europäischen Heteroptera. Er erwarb Material aus Spanien von Staudinger, Apetz und Keitel, aus Italien und Sardinien von Ghiliani, aus Griechenland von Krüper, aus Sarepta (an der Wolga in Rußland) von Becker, aus der Schweiz und Südeuropa von MeyrDür, aus der Steiermark und Dalmatien von Kahr, aus Island, Schweden und Lappland von Staudinger, Boheman und Keitel und aus Mitteleuropa von Tieffenbach. Auf kleinen viereckigen Etiketten (Fig. 1 und 2) sind nicht nur Fundortangaben, sondern oft auch Geber oder Finder genannt. Den wissenschaftlichen Wert dieser genauen Beschriftung hebt STEIN (1864) in seinem Nachruf auf Baerensprung hervor.

Im Jahresbericht des Berliner Museums von 1864 wird von STEIN unter Punkt 6 vermerkt: „Die v. Baerensprung'sche Wanzen Sammlung in Museum-Kästen übertragen“. Einige Jahre später hat Stein dann offenbar Material ausgesondert, denn er schreibt im Jahresbericht von 1870 „Die v. Baerensprung'schen Hemipteren-Sammlung durchgesehen u. das für das Museum Brauchbare herausgenommen".

Bis etwa 1970 wurde die Baerensprung-Sammlung separat aufbewahrt. Sie ist jetzt in die Heteroptera-Hauptsammlung des Museums für Naturkunde integriert.

\section{Liste der Typen der von Baerensprung beschriebene Heteroptera-Arten}

Baerensprungs Material ist an kleinen, etwa $6 \times 6 \mathrm{~mm}$ großen, quadratischen Schildchen zu erkennen. Darauf findet man neben dem Fundort oft auch Angaben zum Sammler (Fig. 1 und 2). Es ist zu vermuten, dass, sofern kein Sammler auf dem Etikett angegeben ist, dieses Material von Baerensprung selbst gesammelt worden ist. Das Typusmaterial von Baerensprung ist nicht immer eindeutig gekennzeichnet. Ein rotes gedrucktes Etikett "Typus“ ist erst später hinzugefügt worden. Diese Kennzeichnung ist außerdem nicht immer vollständig. Da es früher üblich war, in Serien nur das erste Tier mit einem Etikett zu versehen, sind die Etiketten erst später angefertigt worden, sofern sie fehlten. Deshalb ist es möglich, das sich darunter auch Typusexemplare befinden. In der Originalbeschreibung ist manchmal ein Hinweis zu finden, ob der Artbeschreibung ein einzelnes oder mehrere Exemplare zugrunde lagen. 
Das Typusmaterial Baerensprungs wurde oft erst mit der Einordnung in die Sammlung des Museums von STEIN gekennzeichnet und zusätzlich im Sammlungskatalog vermerkt. Außerdem bekam das gesamte Material eine Inventar-Nummer. STEIN hat die Typen

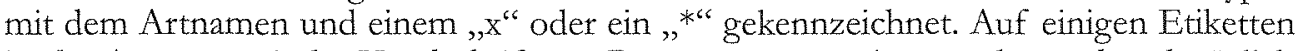
ist der Artname mit der Handschrift von Baerensprung notiert worden und nachträglich von Stein „Baerensprung“" zugefügt worden (Fig. 3). Manchmal stellte sich schon bald nach der Artbeschreibung heraus, dass es sich um ein Synonym handelte. Stein hat dann vermutlich dieses Material gleich zu der Art mit dem gültigen Namen gestellt, so dass die Typusexemplare bis heute unbemerkt blieben.
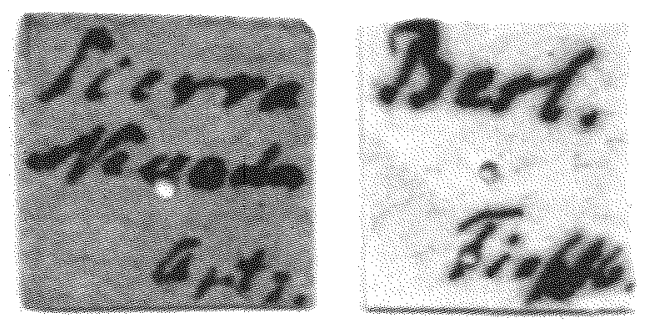

Fig. 1: Fundortetikett mit der Handschrift Baerensprungs "Sierra Nevada Apetz." Dic kleinen Etiketten sind quadratisch und haben Angaben zum Fundort und zum Sammler oder Geber.

Fig. 2: Fundortetikett mit der Handschrift Baerensprungs "Berl. Tieffb." Es handelt sich um Material aus Berlin, das Baerensprung von Tieffenbach erworben hat.

Fig. 3: Dieses Etikett trägt in der oberen Reihe die charakteristische Handschrift von Baerensprung ,maculipennis m.“. Später wurde von Stein „Baerspr. $x^{c \prime}$ hinzugefügt.

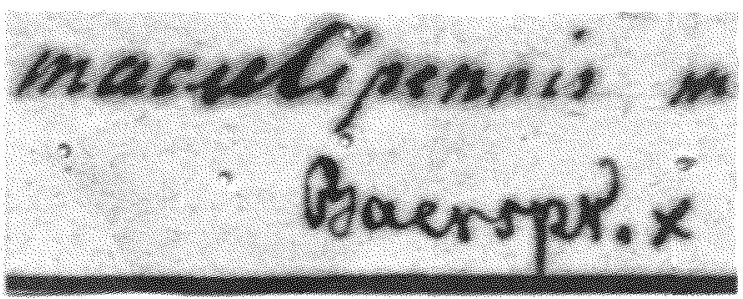

Der Text der Etiketten ist buchstabengenau zitiert. Die Zeilen sind durch einen Schrägstrich, verschiedene Etiketten durch einen doppelten Schrägstrich voneinander getrennt. Zusätzliche Angaben zu den Etiketten sind in eckigen Klammern angefügt. Lectotypen oder Paralectotypen habe ich nur dann festgelegt, wenn es zur eindeutigen Kennzeichnung zur Klärung des Status des Materials notwendig war.

\section{Anthocoridae}

Anthocoris pini BAERENSPRUNG, 1858 (= Elatopbilus pinn)

+ : Berl. // 8571 // Type // Pini Baerenspr. x

Xylocoris rogeri BAERENSPRUNG, 1858 (= Xylocoris cursitans (FALLÉN, 1807) \%: Siles. / [Sammler oder Geber unleserlich] // type // Rogeri / Baerspr. x // 8580 // Xylocoris cursitans Fall. o / J. Péricart det. 1968. Baerensprung gibt verschiedene Fundorte an, darunter auch Berlin. Es ist mehrere zusätzliche Exemplare aus der Sammlung von Baerensprung aus Berlin vorhanden, sie sind mit dem Etikett „Berl.“" versehen, sie könnten möglicherweise auch zur Typusserie von rogeri getechnet werden, eine eindeutige Zuordnung war mir aber nicht möglich. 
Xylocoris cenomyces BAERENSPRUNG, 1858 (= Xyloconis formicetorum (BOHEMAN, 1844)) 2 o 1 \%: Berlin / Tfb. [Die Abkürzung bezieht sich auf den Sammler Tieffenbach] // Typus // Cenomyces / Baerenspr. x. Ein weiteres Exemplar, ein ơ, gehört ebenfalls zur Typusserie: Styria / K[ahr] // Xylocoris formicetorum Boh. / J. Péricart det. 1963. Schließlich ist noch ein o mit den Etiketten: Berl. // coll. Baerensprung [später hinzugefügt] / / Xyloconis formicetorum Boh. / J. Péricart 1967 // Cenomyces m. vorhanden. Ein viertes Typusexemplar ist verloren gegangen. Es ist nur die Nadel mit den Etikett übrig. PÉRICART (1970) erwähnt ein zusätzliches Exemplar aus der Typusserie im Museum in Helsinki.

Xylocoris formicetorum BAERENSPRUNG, 1858 (= Xylocoris formicetorum (BOHEMAN, 1844))

$\sigma^{\pi}$ : Berl. // Coll. Baerensprung [später hinzugefügtes Etikett] // Typus // formicetorum $\mathrm{m}$. [später hinzugefügt mit blauer Tinte:] (nec Boheman) // Xylocoris / formicetorum Boh. / J. Péricart det. 67.

Xylocoris maculipennis BAERENSPRUNG, 1858

$\sigma^{*}$ : Sierra Nevada / Apetz // typus // 8584 // maculipennis m / Baerspr. x

\section{Aradidae}

Aradus pictus BAERENSPRUNG, 1859

Lectotypus (o): Graec. / Krp. // 8805 // pictus v. Baerenspr. * // Typus // Aradus pictus / Baerensprung // design. Vás. 1987, 4 Parelectotypen mit gleichen Daten: Graec. / Krp. (siehe VÁSÁRHELYI 1998)

\section{Lygaeidae}

Auf detaillierte Angaben zu den Lygaeidae (s. 1.) habe ich verzichtet, da sie bereits bei PÉRICART (1998 und 1994) zu finden sind.

Plociomerus lucbsii BAERENSPRUNG, 1859 (= Ligyrocoris syluestris (LINNEAUS, 1758))

(synonymisiert von BAERENSPUNG 1860)

Placiomerus leptopoides BAERENSPRUNG, 1859 (= Paraparomius leptopoides)

Zusätzlich zu dem Lectotypus gibt es ist ein weiteres Exemplar aus Frankreich aus der Sammlung Baerensprung. Obwohl es als Typus gekennzeichnet ist, handelt es sich nicht um Typusmaterial, da Andalusien als locus typicus angegeben worden ist.

Plociomerus collaris BAERENSPRUNG, 1859 (= Pacbybrachius fracticollis (SCHULING, 1829))

Plociomerus annulipes BAERENSPRUNG, 1859 (= Remaudiereana annulipes)

Beosus aeniceps BAERENSPRUNG, 1859 (= Lasiocoris anomalus (KOLENATI, 1845))

Micropus blissoides BAERENSPRUNG, 1859 (= Dimorphopterus blissoides)

Rhyparochromus ibericus BAERENSPRUNG, 1858

Rhyparochromus scaphula BAERENSPRUNG, 1858 (= Ischnopeza birticornis (HERRICHSCHAEFFER, 1850)) 


\section{Pterotmetus crassicornis BAERENSPRUNG, 1858 (= Notochilus crassicornis)}

PÉRICART (1998) gibt an, dass der Typus von crassicornis verloren gegangen ist. Es sind 2 Typusexemplare in der Berliner Sammlung vorhanden, mit folgender Etikettierung: Hisp. / Std.[Staudinger] // Typus // 8519 // mitellatus Costa / crassicornis / v. Baerspr. x // Pterotmetus / crassicornis / Bärensprung / Lectotype o + J. Pericart design. 1993. / Zool. Mus. / Berlin. Das zweite Exemplar ist wie folgt bezettelt: Hisp. / Std. [Staudinger] // Typus // Pterotmetus / crassicornis / Bärensprung / Paralectotype o / J. Pericart design. 1993. // Zool. Mus. / Berlin.

Von einem weiteren Exemplar aus der Typussetie sind nur noch die Femora übriggeblieben.

\section{Alydidae}

Alydus sareptanus BAERENSPRUNG, 1859 (= Megalotomus omaticeps STÅL, 1858)

Unter einem Exemplar ist ein Originaletikett "Sarepta.Becker" und ein zweites Etikett mit der gedruckten Inventarnummer „8934" vorhanden. Dem Inventarbuch ist zu entnehmen, dass es sich dabei um 4 Exemplare aus der Sammlung Baerensprung handelt. Nur ein Exemplar trägt ein Originaletikett, drei weitere waren ursprünglich aus der gleichen Serie, aber ohne eigenes Etikett. Später sind sie beschriftet worden mit „Sarepta Becker Nr. 8934". Auf dem Originaletikett ist mit einem Bleistift ,ornaticeps Stål“ hinzugefügt worden. Aufgrund der Übereinstimmung der Fundortangaben und der eindeutigen Zuordnung zur Sammlung Baerensprung handelt es sich um die Typenserie. Zum Zeitpunkt der Finordnung in die Sammlung des Museums war bereits die Synonymie mit der von Stål beschriebenen ornaticeps bekannt und die ungekennzeichneten Typusexemplare ornaticeps zugeordnet worden. Lectotypus ( $\sigma^{\top}$ ) (hiermit festgelegt): Sarepta Becker (mit schwarzer Tinte, handschriftlich, Originalbeschriftung von Baerensprung) ornaticeps Stål (handschriftlich mit Bleistift) // 8934 // Lectotypus ( ${ }^{7}$ ) / Alydus sareptanus Baerensprung 1858 / J. Deckert design. 2000 / Paralectotypen (hiermit festgelegt) (1 o, 2 우 우): Sarepta / Becker / Nr. 8934 // Paralectotypus / Alydus sareptanus / J. Deckert design. 2000.

\section{Microphysidae}

Anthocoris elegantulus BAERENSPRUNG, 1858 (= Loricula elegantula)

Lectotypus (o): Type // Berl. // 8569// Lecto-Holotype [etikettiert von Péricart 1970]. Entgegen der Angabe von PÉRICART (1970) gehört ein weiteres Exemplar, ein 9 , doch zu dem Typusmaterial. Es trägt das Originalfundortetikett „Berl.“ und die nachträgliche

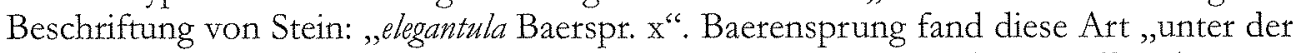
Rinde von Ahorn und Platanen" Ihm lagen also mehrere Exemplare vor. Paralectotypus ( + ) (hiermit festgelegt): Type // Berl. // elegantula Baerspr. x // Anthoconis elegantulus / Baerensprung 1858 Paralectotypus ( $($ ) design. / J. Deckert 2000.

\section{Lichenobia fermiginea BAERENSPRUNG, 1857 (= Ceratocombus coleoptratus SIGNORET, 1852)}

Der Typus ist nicht mehr auffindbar. Es gibt zwar Material in der Sammlung aus Krefeld, das BAERENSPRUNG (1958) dieser Art zugeordnet hatte. Sie ist wie folgt etikettiert: muscorum / Fall. / L. ferruginea / Baerenspr. // Ceratocombus / Signoret. / Licbenobia / Baerenspr. Die Beschreibung der Art bezog jedoch sich auf zwei Exemplare aus der Umgebung von Berlin. 
Myrmedobia rufoscutellata BAERENSPRUNG, 1857 (= Loricula rufoscutellata)

क: Type // 4090 // Kellberg / an faulen / Schwämmen / sehr seltene / Wanze / mit ganzen / Flügeldecken / W. [alt]] // rufo-scutellata / v. Baerenspr. / *. Dem einzigen Typusexemplar fehlt der Kopf.

\section{Miridae}

Teratocoris notatus BAERENSPRUNG, 1859 (= Teratoconis antennatus BOHEMAN, 1852) 9: Dalmat.[ien] / K[a]hr. // 8592 // Typus / / notatus / Baerenspr. / x // Teratocoris / antennatus / Boh. o var. / ( = notatus Bär). Das ist das einzige vorhandene Typusexemplar, dem außerdem der Hinterleib fehlt.

Gryllocoris angusticollis BAERENSPRUNG, 1859 (= Aphanosoma italicum COSTA, 1841) Es sind 8 Syntypen vorhanden, alle mit einem gelben Schildchen: Graec. / Krp. [Krp. = KRÜPER]. Nur ein Exemplar hat zusätzlich zwei Bestimmungsetiketten: angusticollis / Baerensp. x // Gryllocoris / Baerenspr. Die anderen Syntypen haben Originaletiketten mit den Fundortangaben und nachträglich angebrachte gedruckte und auf Fotopapier abgezogene Schildchen:Typus // coll. / Baerensprung.

\section{Pentatomidae}

Eusarcoris angustatus BAERENSPRUNG, 1859 (= Crypsinus angustatus)

In der Sammlung befinden sich 4 Exemplare aus Sarepta, die von Becker gesammelt worden sind und zur Sammlung Baerensprung gehören, von denen drei zu dem 'Typusmaterial von Baerensprung gehören. Sie tragen alle das handschriftliche Etikett von Baerensprung. Das andere Tiere wurden ebenfalls von Becker in Sarepta gesammelt, sind jedoch später inventarisiert worden und müssen nicht unbedingt zur Typusserie zählen. Die drei Syntypen (1 o, 2 o o haben folgende Beschilderung: Cr. angu- / status / 8425 // Sarepta // Sarepta [Originaletikett von BAERENSPRUNG] // Crypsinus angustatus // Typus // Zool. Mus. / Berlin und das zweite Exemplar ist Sarepta // 8425// Typus // Zool. Mus. / Berlin, das dritte (đ) Sarepta // Typus // Zool. Mus./ Berlin.

Graphosoma oculatum BAERENSPRUNG, 1858 (= Derula flavoguttata MULSANT \& REY, 1856)

Das bisher ungekennzeichnete Typusexemplar und befand sich in der Sammlung unter Derula flavoguttata mit dem charakteristischen kleinen quadratischen Etikett von Baerensprung mit der Bezeichnung „Graec.“ und der Inventarnummer 8385. Fundortabgaben, Beschreibung und Abbildung der Art in BAERENSPRUNG (1858) lassen keinen Zweifel daran, dass es sich um ein Typusexemplar handelt. Es ist außerdem kein weiteres Material in der Sammlung Baerensprungs vorhanden, auf das die Beschreibung zutreffen könnte. Weitere Exemplare konnte ich nicht ausfindig machen, obwohl die Originalbeschreibung sich auf mehrere Exemplare bezieht. Hiermit wird ein Lectotypus festgelegt. Lectotypus (đ): Graec. // 8538// Zool. / Mus. Berlin // Graphosoma oculatum / Baerensprung 1858 / Lectotypus design. / J. Deckert 2000 // Derula flavoguttata M. \& R. det. J. Deckert 2000 . 
Leprosoma inconspicuum BAERENSPRUNG, 1859

o: Sarept. // $8380 / /$ Typus // Zool. Mus. / Berlin // [später hinzugefügt mit schwarzem Kugelschreiber:] Lepr. inconsicuum / Baer. Es ist nur ein einziges Typusexemplar vorhanden.

Strachia lineola BAERENSPRUNG, 1858 (= Eurydema lineola)

o: lineola / Baerenspr. x // Typus // 8/9// Astur.[ien] / StD. // 8447.

Es sind keine weiteren Typusexemplare vorhanden.

\section{Tingidae und Canthacaderidae}

Dictyonota marmorea BAERENSPRUNG, 1858 (Typus nicht auffindbar)

Dictyonota albipennis BAERENSPRUNG, 1858 (Typus nicht auffindbar)

Taphrostethus stauderingeri BAERENSPRUNG, 1858 (= Cantacader quadricornis (LE PELETIER \& AUDINET-SERVILLE, 1828))

Ein Exemplar: Typus / Museum Berlin / Taphrostethus / standingeri / Baerenspung // Andalusien / Staudinger [rotes Schild mit gedrucktem Text, keine Originaletiketten am Exemplar vorhanden.] // Cantacader / quadripunctatus Le Plt\& / J. Péricart, det. 1978.

\section{Baerensprungs entomologische Artikel:}

1. Beobachtungen über einige einheimische Arten aus der Familie der Cocciden. - D' Alton Zeitschrift für Zoologie 1, 1848: 165-170; 173-176.

2. Myrmedobia und Lichenobia, zwei neue einheimische Rhynchoten-Gattungen. - Berliner Entomologische Zeitschrift 1, 1857. [Abb. in Teil II²], 161-168.

3. Synonymische Bemerkungen. Ueber Hemiptera. - Berliner Entomologische Zeitschrift 1858, 2: 79-81.

4. Neue und seltene Rhynchoten der europäischen Fauna. - Berliner Entomologische Zeitschrift 1858, 2, 188-208, Taf. II.

5. Neue und seltene Rhynchoten der europäischen Fauna. Zweites Stück (im Anschluß an Jahrgang II.). - Berliner Entomologische Zeitschrift 1859, 3, 329-338, Taf. VI.

6. Hemiptera Heteroptera Europaea systematice disposa. - Berliner Entomologische Zeitschrift 1860, 4 : Appendix 1-25.

7. Catalogus Hemipterorum Europae. Berlin 8. , 1-25. [Separatabdruck der Arbeit 6 ]

\section{Danksagung}

Herrn J. FISCHER (Dabendorf) danke ich für die Beschaffung des Artikels über Baerensprung aus den Annalen des Charité-Krankenhauses.

\footnotetext{
${ }^{2}$ Im Bd. 2 der Berliner Entomologischen Zeitschrift von 1858 ist Myrmedobia auf Tf. II. abgebildet.
} 


\section{Literatur}

HAGEN, H. A. 1862: Bibliotheca Entomologica. Die Litteratur über das ganze Gebiet der Entomologie bis zum Jahre 1862. - Band 1, Leipzig, I-XII: 1-566.

HORN, W; KAHLE, I.; FRIESE, G. \& GAEDIKE, R. 1990: Collectiones entomologicae. Ein Kompendium über den Verbleib entomologischer Sammlungen der Welt bis 1960, Teil I: A bis K. - Akademie der Landwirtschaftswissenschaften der DDR: 1-220.

PÉRICART, J. 1970: Désignation de néotypes, lectotypes et paralectotypes pour diverses espèces paléarctiques d'Anthocoridae et Microphysidae [Hemiptera]. - Annales Société entomologique de France (N. S.) 6 (3): 733-755.

PÉRICART, J. 1994: Les Drymini euro-méditerranéens Notes systématiques et synonymiques (Heteroptera, Lygaeidae). - Bulletin de la Société entomologique de France 99 (4): 403-418.

PÉRICART, J. 1998: Désignation de lectotypes et paralectotypes pour des Lygaeidae paléarctiques et commentaires [Heteroptera] 5. Les Types des Auteurs Allemands, Italiens et d'auteurs divers. - Revue française d' entomologie (N. S.) 20 (1-2): 5-12.

SCHUMACHER, F. 1912a: Die Rhynchoten-Fauna der Mark Brandenburg. II. Coreidae. - Berliner Entomologische Zeitschrift LVI: 128-136.

SCHUMACHER, F. 1912b: Die Rhynchoten-Fauna der Mark Brandenburg. IV. Fam. Berytidae (Neididae). Berliner Entomologische Zeitschrift LVII: 131-143.

SCHUMAChER, F. 1913: Verzeichnis der Wanzen, welche F. v. BAERENSPRUNG bei Halle beobachtet hat. Archiv für Naturgeschichte Berlin 79, Abteilung A, Heft 3: 87-91.

STEIN, J. P. E. F. 1864: Des verst. Professors v. BAERENSPRUNG hinterlassene Hemipteren-Sammlung. Berliner Entomologische Zeitschrift 8: 383-384.

VÁSÁRHELYI, T. 1998: New Palaearctic Aradus species in the betulae-group (Heteroptera, Aradidae). - Annales historico-naturales Museu nationalis hungarici 80: 57-63.

VEIT, O. 1865: Zur Erinnerung an Professor Dr. FEIIX YoN BÄRENSPRUNG. - Annalen des Chatité-Krankenhauses und der übrigen Königlichen medicinischen-chirurgischen Leht- und Kranken-Anstalten zu Berlin XII, Heft 2: 75-85.

\section{Anschrift des Verfassers:}

\section{Dr. JÜRGEN DECKERT}

Museum für Naturkunde der Humboldt-Universität zu Berlin

Institut für Systematische Zoologie

Invalidenstraße 43

D-10115 Berlin

Deutschland

e-mail: juergen.deckert@rz.hu-berlin.de 